\title{
Commentary: Novel Cell Culture Paradigm Prolongs Mouse Corneal Epithelial Cell Proliferative Activity In Vitro and In Vivo
}

\author{
Xiuna $\mathrm{Ji}^{1}$, Mingyue Zheng ${ }^{2}$, Tingjun Fan ${ }^{1}$ and Bin $\mathrm{Xu}^{1 \text { * }}$ \\ ${ }^{1}$ College of Marine Life Sciences, Ocean University of China, Qingdao, China, ${ }^{2}$ Guangdong Provincial People's Hospital, \\ Guangdong Academy of Medical Sciences, Guangzhou, China
}

Keywords: limbal stem cells, self-renewal, in vitro expansion, small-molecule compounds, regenerative medicine

\section{A Commentary on}

Novel Cell Culture Paradigm Prolongs Mouse Corneal Epithelial Cell Proliferative Activity In Vitro and In Vivo

by An X., Wang G., Jin M., Zhou X., Gao S., Chen J., Reinach P. S., Liu Z., Xue Y., and Li C. (2021). Front. Cell Dev. Biol. 9:675998. doi: 10.3389/fcell.2021.675998

\section{OPEN ACCESS}

Edited by:

Valerie Kouskoff,

The University of Manchester,

United Kingdom

Reviewed by:

Hong Ouyang,

Sun Yat-sen University, China

${ }^{*}$ Correspondence:

$\operatorname{Bin} X u$

bxu@ouc.edu.cn

Specialty section:

This article was submitted to

Stem Cell Research,

a section of the journal

Frontiers in Cell and Developmental

Biology

Received: 26 November 2021

Accepted: 19 January 2022

Published: 18 February 2022

\section{INTRODUCTION}

Limbal stem cells (LSCs) are located in the limbal palisades of Vogt between the cornea and conjunctiva. The LSCs exhibit a series of key characteristics of epithelial stem cells, including selfrenewal ability, high proliferation potential, and tissue regeneration capacity (Li et al., 2021a). The loss of LSCs and damage to their microenvironment contribute to various corneal diseases such as LSC deficiency (LSCD). LSCD further causes corneal vascularization, opacification, and even blindness (Singh and Sangwan, 2021). Better treatments for LSCD are currently being developed, including LSC culture for autograft. In 2015, Holoclar [ex vivo expanded autologous human corneal epithelial cells (CECs) containing stem cells] successfully gained the marketing authorization from the European Medicines Agency to treat moderate-to-severe LSCD following chemical and thermal eye burns (Yu et al., 2018). The regenerative potential of Holoclar mainly relies upon the highly proliferative and self-renewing properties of holoclones. However, it still needs to be further explored on how to optimize LSC expansion in vitro. In fact, current efforts are focused on the addition of small molecular compounds to improve efficiency of stem cell expansion. These compounds have clear targets, show quick action and reversibility, and are therefore of great value in the research and practical application toward maintaining LSC self-renewal.

\section{A NOVEL STRATEGY TO OPTIMIZE SELF-RENEWAL AND EXPANSION OF LIMBAL STEM CELLS IN CULTURE}

It is well known that increasing extracellular calcium, serum and air-lifting can lead to terminal differentiation and gradual loss of LSCs (Meyer-Blazejewska et al., 2010). Previously, Xiang et al. has reported the long-term in vitro maintenance of primary human hepatocytes by modulating cell signaling pathways with a combination of five chemicals (5C), including forskolin, SB431542, DAPT, 


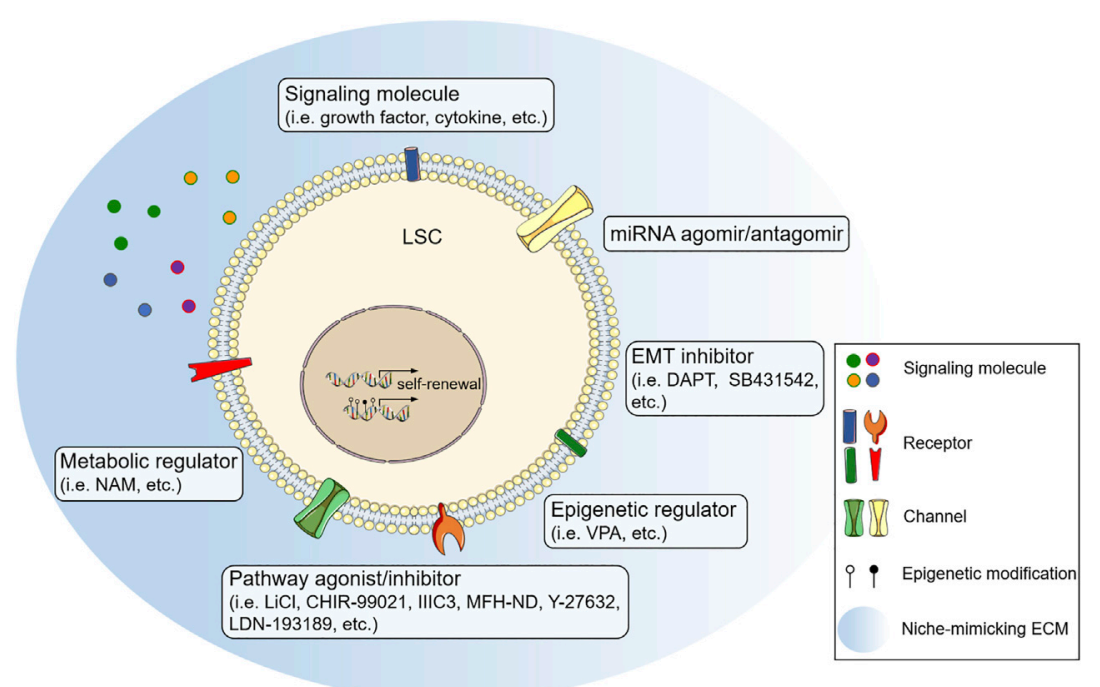

FIGURE 1 | Small-molecular combination as a novel strategy to optimize limbal stem cell (LSC) expansion in culture. GSK3 $\beta$ inhibitors [lithium chloride (LiCl), CHIR99021], DKK inhibitor (IIIC3), and Wnt mimic (MFH-ND) activate the canonical Wnt pathway to improve LSC expansion. Growth factor (pigment epithelial-derived factor), cytokine (leukemia inhibitory factor), ROCK inhibitor (Y-27632), and BMP signaling inhibitor (LDN-193189) also promote LSC self-renewal through multiple signaling pathways. TGF/ $\beta$ signaling inhibitor (SB431542) and Notch signaling inhibitor (DAPT) prevent epithelial-mesenchymal transition of LSC by inhibiting the corresponding pathways. Histone deacetylase inhibitor (VPA), miRNA agomir/antagomir and nicotinamide adenine dinucleotide (NAD) precursor [nicotinamide (NAM)] might enhance the efficiency of LSC expansion through the regulation at epigenetic, post-transcriptional, and metabolic levels. ECM, extracellular matrix; EMT, epithelialmesenchymal transition; miRNA, microRNA.

IWP-2, and LDN-193189 (Xiang et al., 2019). Interestingly, each compound of $5 \mathrm{C}$ has been proved to improve corneal epithelium homeostasis. This study by An et al. (2021) compared the effects of 5C and 6C (combined Y-27632 and 5C) on the mouse CECs (mCECs) and found that $6 \mathrm{C}$ could increase mCEC proliferation, sustain the expression levels of the progenitor cell function gene, as well as suppress epithelial-mesenchymal transition. The $6 \mathrm{C}$ culture method may be applied for improving the availability of CECs to treat LSCD in clinical practice.

The 6C improve the maintenance of mCEC morphology and function in long-term culture, subculture in vitro, and mouse cornea culture ex vivo. Moreover, the $6 \mathrm{C}$ culture system conduces to construct tissue-engineered corneal epithelium and promotes healing of corneal epithelial wounds in mice. These smallmolecule combinations regulate $\mathrm{mCEC}$ proliferation involving in cAMP, TGF- $\beta$, BMP, Notch, Wnt/ $\beta$-catenin, and Rho/ROCK signaling (An et al., 2021). Remarkably, 6C seem to maintain the limbal proliferating stem and progenitor cell phenotype in vivo, which demonstrates that these signaling modulators might regulate LSC functions. Considering that CECs have a finite capacity to replicate and eventually enter irreversible growth arrest, it is more significant to apply this novel strategy to LSC expansion based on the mechanisms underlying LSC selfrenewal. Then, specific small-molecule compounds can be optimized to act on the signaling regulatory network for LSC expansion in culture.

Transcription factor (TF) PAX6 is expressed during eye development, which is considered as the master gene for oculogenesis (Ramos et al., 2015). PAX6 plays an essential role in specifying LSCs, in which Wnt7a controls CEC fate determination through PAX6 (Ouyang et al., 2014). GSK3 $\beta$ inhibitors, lithium chloride, and CHIR-99021 can activate the canonical Wnt pathway to improve LSC self-renewal (Bonnet et al., 2021). Specific small molecules such as IIIC3 (DKK inhibitor) and MFH-ND (Wnt mimic) have been designed to improve LSC expansion in vitro by interacting specifically with the Wnt co-receptors LRP5/6 and FZD (Janda et al., 2017; Gonzalez et al., 2019; Chen et al., 2020; Zhang et al., 2020). TFs RUNX1 and SMAD3 are also required for maintenance of corneal epithelial identity and homeostasis by interactions with PAX6 (Li et al., 2021c). Moreover, RUNX1 can shape LSC chromatin architecture via modulating $\mathrm{H} 3 \mathrm{~K} 27 \mathrm{ac}$ deposition ( $\mathrm{Li}$ et al., 2021c). RNA sequencing (RNA-seq) and qualitative proteomics identify that miR146a has an opposite regulatory role in the fine-tuning of Notch $1 / 2$ expression to balance LSC self-renewal and differentiation (Poe et al., 2020). The above studies suggest that LSC self-renewal is regulated by a variety of signaling pathways ranging from signaling factor, TF, epigenetic regulator to microRNA, which serve as putative targets of smallmolecule compounds during LSC culture.

LSCs certainly need to communicate with their own niche to maintain self-renewal ( $\mathrm{Li}$ et al., 2007), including specific extracellular matrix (ECM), niche cells, and signaling molecules (Ashworth et al., 2021). The ECM not only anchors the basal epithelium but also mediates intercellular communication and provides distinct mechanical properties that influence LSC phenotype, population, and self-renewal (Gesteira et al., 2017; Gouveia et al., 2019; Zheng et al., 2019; Zhu et al., 2020; Ashworth et al., 2021). Additionally, cell-cell communication analysis reveals the central role of LSCs and their 
bidirectional regulation with various niche cells, such as limbal-mesenchymal stem cells, $\mathrm{CD}_{4} 5^{+}$cells, $\mathrm{PAX}^{+}$cells, and melanocytes (Yazdanpanah et al., 2019; Altshuler et al., 2021; Chen et al., 2021; Polisetti et al., 2021). The surrounding niche cells regulate LSC homeostasis by modulating signaling pathways such as Wnt, Notch, TGF- $\beta$, and BMP signaling (Bonnet et al., 2021). These indicate that LSC culture surface needs to satisfy the component and stiffness of limbal niche to regulate the signaling network of LSC self-renewal which might be used as effective targets of specific small molecules. Significantly, it is now wellaccepted that culturing cells in three-dimensional systems that mimic key factors of tissues is much more representative of the in vivo microenvironment than simple two-dimensional monolayers (Langhans, 2018). Therefore, the small-molecule application shows its advantage for LSC expansion in a nichemimicking culture system via cellular signaling crosstalk.

Single-cell RNA-seq (scRNA-seq) technologies are broadly applied to dissect the cellular heterogeneity and expression dynamics, providing unprecedented insights into single-cell biology (Plass et al., 2018; Li et al., 2020). A few groups have recently used scRNA-seq to analyze LSC populations and corneal homeostasis (Li et al., 2021a; Li et al., 2021b). These data provide a new avenue for studying the mechanisms of LSC self-renewal (Altshuler et al., 2021). Single-cell transcriptomics have identified that TFs (TP63, MYC, PARP1, and SOX17) have important roles in regulating LSC stemness and proliferation (Li et al., 2021a; Dou et al., 2021). Moreover, two novel surface markers (GPHA2 and TSPAN7) support LSC self-renewal (Li et al., 2021a; Collin et al., 2021), suggesting that $\mathrm{GPHA}^{+}$and/or TSPAN7 ${ }^{+}$LSCs can be isolated from multiple cell types in limbus by flow cytometry and cell sorting technology. Significantly, thioredoxin-interacting protein (TXNIP), a metabolic protein involved in redox regulation, is highly expressed in LSCs, which may be a novel LSC-preferred gene that contributes to stem cell maintenance (Kaplan et al., 2019). It also indicates that there might be a close relationship between cellular metabolism and LSC self-renewal. The combination of single-cell transcriptomics

\section{REFERENCES}

Altshuler, A., Amitai-Lange, A., Tarazi, N., Dey, S., Strinkovsky, L., Hadad-Porat, S., et al. (2021). Discrete Limbal Epithelial Stem Cell Populations Mediate Corneal Homeostasis and Wound Healing. Cell Stem Cell 28, 1248-1261. doi:10.1016/j.stem.2021.04.003

An, X., Wang, G., Jin, M., Zhou, X., Gao, S., Chen, J., et al. (2021). Novel Cell Culture Paradigm Prolongs Mouse Corneal Epithelial Cell Proliferative Activity In Vitro and In Vivo. Front. Cel Dev. Biol. 9, 675998. doi:10.3389/fcell.2021. 675998

Ashworth, S., Harrington, J., Hammond, G. M., Bains, K. K., Koudouna, E., Hayes, A. J., et al. (2021). Chondroitin Sulfate as a Potential Modulator of the Stem Cell Niche in Cornea. Front. Cel Dev. Biol. 8, 567358. doi:10.3389/ fcell.2020.567358

Bonnet, C., González, S., Roberts, J. S., Robertson, S. Y. T., Ruiz, M., Zheng, J., et al. (2021). Human Limbal Epithelial Stem Cell Regulation, Bioengineering and Function. Prog. Retin. Eye Res. 85, 100956. doi:10.1016/j.preteyeres.2021. 100956

Chen, H., Lu, C., Ouyang, B., Zhang, H., Huang, Z., Bhatia, D., et al. (2020). Development of Potent, Selective Surrogate WNT Molecules and Their Application in Defining Frizzled Requirements. Cel Chem. Biol. 27, 598-609. e4. doi:10.1016/j.chembiol.2020.02.009 and metabolomics appears to help us comprehensively understand the mechanisms of LSC self-renewal. And these multi-omics analysis will provide inspiring evidence to develop small-molecule compounds for LSC expansion.

\section{CONCLUSION}

The long-term maintenance of cell function requires a sophisticated signaling regulatory network; a chemical strategy using small-molecule combinations confers the advantage of synergistically orchestrating innate signals to achieve spatiotemporal modulations of specific cellular targets (Xu et al., 2015). This is the first report on the 6C culture system prolonging $\mathrm{mCEC}$ maintenance in vitro. The chemical approach is simple and easily applied for autologous epithelial sheet transplantation. It also provides a new idea and method for LSC expansion in vitro (Figure 1). More stable LSC populations can be obtained for applications in regenerative medicine research by optimizing specific small-molecule combinations under a niche-mimicking culture condition.

\section{AUTHOR CONTRIBUTIONS}

BX conceived the article. XJ and BX wrote the first draft. MZ, TF, and $\mathrm{BX}$ reviewed and revised the manuscript. All authors contributed to the article and approved the submitted version.

\section{FUNDING}

This work was supported by grants from the National Natural Science Foundation of China (31601104) and Natural Science Foundation of Shandong Province (ZR2021MC158).

Chen, S.-Y., Zhu, Y., Zhang, Y., Hsu, D., and Tseng, S. C. G. (2021). HC-HA/PTX3 from Amniotic Membrane Reverts Senescent Limbal Niche Cells to Pax6+ Neural Crest Progenitors to Support Limbal Epithelial Progenitors. Stem Cells 39, 280-295. doi:10.1002/stem.3323

Collin, J., Queen, R., Zerti, D., Bojic, S., Dorgau, B., Moyse, N., et al. (2021). A Single Cell Atlas of Human Cornea that Defines its Development, Limbal Progenitor Cells and Their Interactions with the Immune Cells. Ocul. Surf. 21, 279-298. doi:10.1016/j.jtos.2021.03.010

Dou, S., Wang, Q., Qi, X., Zhang, B., Jiang, H., Chen, S., et al. (2021). Molecular Identity of Human Limbal Heterogeneity Involved in Corneal Homeostasis and Privilege. Ocul. Surf. 21, 206-220. doi:10.1016/j.jtos.2021.04.010

Gesteira, T. F., Sun, M., Coulson-Thomas, Y. M., Yamaguchi, Y., Yeh, L.-K., Hascall, V., et al. (2017). Hyaluronan Rich Microenvironment in the Limbal Stem Cell Niche Regulates Limbal Stem Cell Differentiation. Invest. Ophthalmol. Vis. Sci. 58, 4407-4421. doi:10.1167/iovs.17-22326

González, S., Oh, D., Baclagon, E. R., Zheng, J. J., and Deng, S. X. (2019). Wnt Signaling Is Required for the Maintenance of Human Limbal Stem/progenitor Cells In Vitro. Invest. Ophthalmol. Vis. Sci. 60, 107-112. doi:10.1167/iovs.1825740

Gouveia, R. M., Vajda, F., Wibowo, J. A., Figueiredo, F., and Connon, C. J. (2019). YAP, $\triangle \mathrm{Np} 63$, and $\beta$-Catenin Signaling Pathways Are Involved in the Modulation of Corneal Epithelial Stem Cell Phenotype Induced by Substrate Stiffness. Cells 8, 347. doi:10.3390/cells8040347 
Janda, C. Y., Dang, L. T., You, C., Chang, J., de Lau, W., Zhong, Z. A., et al. (2017). Surrogate Wnt Agonists that Phenocopy Canonical Wnt and $\beta$-catenin Signalling. Nature 545, 234-237. doi:10.1038/nature22306

Kaplan, N., Wang, J., Wray, B., Patel, P., Yang, W., Peng, H., et al. (2019). Singlecell RNA Transcriptome Helps Define the Limbal/corneal Epithelial Stem/early Transit Amplifying Cells and How Autophagy Affects This Population. Invest. Ophthalmol. Vis. Sci. 60, 3570-3583. doi:10.1167/iovs.19-27656

Langhans, S. A. (2018). Three-dimensional In Vitro Cell Culture Models in Drug Discovery and Drug Repositioning. Front. Pharmacol. 9, 6. doi:10.3389/fphar. 2018.00006

Li, W., Hayashida, Y., Chen, Y.-T., and Tseng, S. C. (2007). Niche Regulation of Corneal Epithelial Stem Cells at the Limbus. Cell Res 17, 26-36. doi:10.1038/sj. cr.7310137

Li, Y., Xu, Q., Wu, D., and Chen, G. (2020). Exploring Additional Valuable Information from Single-Cell RNA-Seq Data. Front. Cel Dev. Biol. 8, 593007. doi:10.3389/fcell.2020.593007

Li, D.-Q., Kim, S., Li, J.-M., Gao, Q., Choi, J., Bian, F., et al. (2021a). Singlecell Transcriptomics Identifies Limbal Stem Cell Population and Cell Types Mapping its Differentiation Trajectory in Limbal Basal Epithelium of Human Cornea. Ocul. Surf. 20, 20-32. doi:10.1016/j.jtos. 2020.12.004

Li, J.-M., Kim, S., Zhang, Y., Bian, F., Hu, J., Lu, R., et al. (2021b). Single-cell Transcriptomics Identifies a Unique Entity and Signature Markers of TransitAmplifying Cells in Human Corneal Limbus. Invest. Ophthalmol. Vis. Sci. 62, 36. doi:10.1167/iovs.62.9.36

Li, M., Huang, H., Li, L., He, C., Zhu, L., Guo, H., et al. (2021c). Core Transcription Regulatory Circuitry Orchestrates Corneal Epithelial Homeostasis. Nat. Commun. 12, 420. doi:10.1038/s41467-020-20713-z

Meyer-Blazejewska, E. A., Kruse, F. E., Bitterer, K., Meyer, C., Hofmann-Rummelt, C., Wünsch, P. H., et al. (2010). Preservation of the Limbal Stem Cell Phenotype by Appropriate Culture Techniques. Invest. Ophthalmol. Vis. Sci. 51, 765-774. doi:10.1167/iovs.09-4109

Ouyang, H., Xue, Y., Lin, Y., Zhang, X., Xi, L., Patel, S., et al. (2014). WNT7A and PAX6 Define Corneal Epithelium Homeostasis and Pathogenesis. Nature 511, 358-361. doi:10.1038/nature13465

Plass, M., Solana, J., Wolf, F. A., Ayoub, S., Misios, A., Glažar, P., et al. (2018). Cell Type Atlas and Lineage Tree of a Whole Complex Animal by Single-Cell Transcriptomics. Science 360, eaaq1723. doi:10.1126/science.aaq1723

Poe, A. J., Kulkarni, M., Leszczynska, A., Tang, J., Shah, R., Jami-Alahmadi, Y., et al. (2020). Integrated Transcriptome and Proteome Analyses Reveal the Regulatory Role of miR-146a in Human Limbal Epithelium via Notch Signaling. Cells 9, 2175. doi:10.3390/cells9102175

Polisetti, N., Giessl, A., Zenkel, M., Heger, L., Dudziak, D., Naschberger, E., et al. (2021). Melanocytes as Emerging Key Players in Niche Regulation of Limbal Epithelial Stem Cells. Ocul. Surf. 22, 172-189. doi:10.1016/j.jtos.2021.08.006
Ramos, T., Scott, D., and Ahmad, S. (2015). An Update on Ocular Surface Epithelial Stem Cells: Cornea and Conjunctiva. Stem Cell Int. 2015, 1-7. doi:10.1155/2015/601731

Singh, A., and Sangwan, V. S. (2021). Mini-review: Regenerating the Corneal Epithelium with Simple Limbal Epithelial Transplantation. Front. Med. 8, 673330. doi:10.3389/fmed.2021.673330

Xiang, C., Du, Y., Meng, G., Soon Yi, L., Sun, S., Song, N., et al. (2019). Long-term Functional Maintenance of Primary Human Hepatocytes In Vitro. Science 364, 399-402. doi:10.1126/science.aau7307

Xu, J., Du, Y., and Deng, H. (2015). Direct Lineage Reprogramming: Strategies, Mechanisms, and Applications. Cell Stem Cell 16, 119-134. doi:10.1016/j.stem. 2015.01 .013

Yazdanpanah, G., Haq, Z., Kang, K., Jabbehdari, S., Rosenblatt, M. 1., and Djalilian, A. R. (2019). Strategies for Reconstructing the Limbal Stem Cell Niche. Ocul. Surf. 17, 230-240. doi:10.1016/j.jtos.2019.01.002

Yu, T. T. L., Gupta, P., Ronfard, V., Vertès, A. A., and Bayon, Y. (2018). Recent Progress in European Advanced Therapy Medicinal Products and beyond. Front. Bioeng. Biotechnol. 6, 130. doi:10.3389/fbioe.2018.00130

Zhang, C., Mei, H., Robertson, S. Y. T., Lee, H.-J., Deng, S. X., and Zheng, J. J. (2020). A Small-Molecule Wnt Mimic Improves Human Limbal Stem Cell Ex Vivo Expansion. iScience 23, 101075. doi:10.1016/j.isci.2020.101075

Zheng, M., Tian, C., Fan, T., and Xu, B. (2019). Fibronectin Regulates the Self-Renewal of Rabbit Limbal Epithelial Stem Cells by Stimulating the Wnt11/Fzd7/ROCK Noncanonical Wnt Pathway. Exp. Eye Res. 185, 107681. doi:10.1016/j.exer.2019.05.021

Zhu, J., Wang, L.-y., Li, C.-y., Wu, J.-y., Zhang, Y.-t., Pang, K.-p., et al. (2020). SPARC Promotes Self-Renewal of Limbal Epithelial Stem Cells and Ocular Surface Restoration through JNK and P38-MAPK Signaling Pathways. Stem Cells 38, 134-145. doi:10.1002/stem.3100

Conflict of Interest: The authors declare that the research was conducted in the absence of any commercial or financial relationships that could be construed as a potential conflict of interest.

Publisher's Note: All claims expressed in this article are solely those of the authors and do not necessarily represent those of their affiliated organizations, or those of the publisher, the editors and the reviewers. Any product that may be evaluated in this article, or claim that may be made by its manufacturer, is not guaranteed or endorsed by the publisher.

Copyright (c) $2022 \mathrm{Ji}$, Zheng, Fan and Xu. This is an open-access article distributed under the terms of the Creative Commons Attribution License (CC BY). The use, distribution or reproduction in other forums is permitted, provided the original author(s) and the copyright owner(s) are credited and that the original publication in this journal is cited, in accordance with accepted academic practice. No use, distribution or reproduction is permitted which does not comply with these terms. 